\title{
EL ASEGURAMIENTO DE LA CALIDAD EN EL INSTITUTO CONSORCIO CLAVIJERO
}

\author{
(QUALITY ASSURANCE AT THE CONSORCIO CLAVIJERO INSTITUTE)
}

\author{
Ricardo Mercado del Collado \\ Mónica López Granados \\ Gustavo Balderas Rosas \\ Instituto Consorcio Clavijero (México)
}

\section{RESUMEN}

En este trabajo se analiza el crecimiento de la matrícula de educación superior mundial en programas a distancia como parte de la estrategia emprendida por los gobiernos para incrementar su competitividad en una economía basada en el conocimiento. Se presenta, además, información sobre la educación a distancia en México, especialmente en su modalidad virtual y se analizan posibles causas de su lenta evolución, para después analizar distintos modelos de aseguramiento de la calidad de la educación a distancia y presentar el modelo empleado en el Instituto Consorcio Clavijero compuesto por los siguientes elementos: pertinencia de la oferta, legalidad de los programas, calidad de los cursos y materiales de aprendizaje, perfil del profesor, acceso a la tecnología, inducción de estudiantes, apoyo estudiantil, desempeño de profesores, aprendizaje, satisfacción de estudiantes, seguimiento de egresados y plataforma tecnológica.

Palabras clave: educación a distancia, modalidad virtual, educación superior, aseguramiento de la calidad.

\begin{abstract}
This article reports our analysis of world enrollment growth in distance education programs promoted by governments to increase their competitiveness in the knowledge economy. Information is presented regarding Mexico's distance education system, specifically its online programs and reviews possible reasons for its slow development. Furthermore, it analyses different quality assurance models and describes the one used at the Consorcio Clavijero Institute to examine the following: relevance, legality, course design quality, teacher profile, technology access, student preparation, student support, teacher support and performance, learning, student satisfaction, alumni follow up and the learning management system.
\end{abstract}

Keywords: distance education, online programs, higher education, quality assurance. 


\section{PAPEL DE LA EDUCACIÓN SUPERIOR EN LA SOCIEDAD DE LA INFORMACIÓN Y EL CONOCIMIENTO}

La educación superior es hoy, más que nunca, motivo de interés prioritario en las agendas nacionales. El interés de los gobiernos por la educación superior se deriva del reconocimiento generalizado sobre su contribución como motor de la competitividad económica de los países, en una economía mundial sustentada de manera creciente en el conocimiento. La OCDE estimaba en 2001 que las industrias basadas en el conocimiento que incluyen a las principales productoras de bienes de alta tecnología, de manufactura con base en alta y media tecnología y de servicios usuarios de tecnología (finanzas, aseguradoras, negocios y comunicaciones) representaban más de la mitad del PIB de las economías más desarrolladas (OCDE, 2001).

A diferencia de las economías prevalecientes durante buena parte del siglo XIX y la primera mitad del siglo XX, donde la razón principal de la expansión económica de los países era la acumulación de capital y trabajo, en la nueva economía del conocimiento es el propio conocimiento y la información lo que se transforma para generar nuevos conocimientos, mismos que se cotizan alto en los mercados internacionales.

A partir de la revisión de diferentes acepciones del concepto de economía del conocimiento, Robles (2006) señala que este tipo de economías comparten los siguientes cinco atributos: 1) capital humano calificado y un uso intensivo del conocimiento en la producción, 2) ser economías atractivas en el entorno internacional con altos niveles de competitividad y una orientación hacia el exterior, 3) un marco institucional y un capital social que favorezcan la certidumbre y la confianza, 4) poseer sistemas de innovación y una capacidad emprendedora, y 5) una infraestructura de información, comunicación y tecnología adecuada y amplia.

Por su parte, la OCDE (2008) identifica la contribución de la educación superior al desarrollo económico y social de las naciones en el logro de los siguientes fines: la formación de capital humano, la construcción de bases de conocimiento, la difusión y uso del conocimiento, y el almacenamiento del conocimiento.

Es clara la relación entonces entre las características que comparten las economías del conocimiento y los propósitos que persiguen, en la actualidad, las instituciones educativas de nivel superior. Dichas instituciones contribuyen en la formación del capital humano cualificado, en la innovación mediante la investigación 
y sus aplicaciones en la producción, así como en el desarrollo y uso extendido de tecnologías de la información y la comunicación.

Debido al valor estratégico que asumen las instituciones de educación superior en el desarrollo de la competitividad de las naciones, se ha impulsado mundialmente el crecimiento de los sistemas de educación superior. Este crecimiento ha tenido lugar en las modalidades escolarizadas como en las modalidades a distancia, y más recientemente, en línea.

\section{LA EDUCACIÓN A DISTANCIA EN LA AMPLIACIÓN DE LA COBERTURA DE EDUCACIÓN SUPERIOR}

La matrícula estudiantil de nivel superior ha tenido un crecimiento considerable en el mundo, especialmente en los últimos años. De acuerdo con datos de la UNESCO para el año 2007 había 152.5 millones de estudiantes en el mundo, con una tasa anual de crecimiento promedio de $4.6 \%$ entre 1970 y 2007 , lo que supone la duplicación de la matrícula cada 15 años (UNESCO, 2009). Este crecimiento ha tenido lugar en las instituciones que ofrecen sus programas en modalidades presenciales, pero también se observa un crecimiento considerable en la participación de las instituciones a distancia.

En los Estados Unidos de América, por ejemplo, la tasa de crecimiento anual de $12.9 \%$ para la educación en línea supera la de $1.2 \%$ en la población global estadounidense de educación superior (Sloan Consortium, 2008). La penetración de la educación a distancia en los sistemas de educación presencial también muestra un crecimiento sostenido en ese país. Se estima que en el otoño de 2009 cerca de 5.6 millones de estudiantes universitarios tomaron un curso en línea, un millón más que en 2008 (Allen y Seaman, 2010). En Canadá, tan sólo la Athabasca University, reporta para el ciclo 2008-2009 una matrícula de 38464 estudiantes con 776 cursos y más de 90 programas de posgrado (Athabasca University, 2009).

En Europa, Reino Unido y España son dos países con avances significativos. La Open University de Inglaterra con más de 100 años de experiencia en educación a distancia, y ahora líder mundial en el uso de las tecnologías de la información y comunicación, reporta una matrícula de 250000 estudiantes que aprenden desde sus hogares por Internet (Open University, 2010). En España, una institución, la Universidad Nacional a Distancia, UNED, reporta 205931 estudiantes (UNED, 2010). 
Los datos de algunos países de Asia y Oceanía son también evidencia del crecimiento vertiginoso de la educación a distancia en el mundo. La Indhira Gandhi National Open University posee una matrícula de 2.6 millones de estudiantes atendidos mediante la combinación de medios que van desde textos impresos a la comunicación asíncrona por Internet (Indhira National Open University, 2010). La Universidad Abierta de China, por su parte, reporta una población activa de 2.7 millones de estudiantes de los cuales 802600 estudian una licenciatura, 1823600 en programas de profesional asociado y 169900 en el programa "un estudiante universitario por una comunidad" (Open University of China, 2010). En Australia, el consorcio llamado Open Universities Australia reporta para 2010 una matrícula de 131000 estudiantes, 36\% más que en 2009 (Open Universities Australia, 2010).

Inclusive África muestra avances en esta materia. La University of South Africa registró una matrícula de 263470 estudiantes en 2009, lo que supone casi una tercera parte de la matrícula de educación superior del país (University of South Africa, 2010).

Para la región de América Latina la información disponible de 2005, indicaba la existencia de 459 instituciones con programas de educación virtual en marcha, representando el 5,5\% del total de 6500 instituciones de educación superior de la región (IESALC, 2009). En términos de matrícula, estimaciones hechas para ese año, calculaban la matrícula en cerca de 165000 estudiantes, lo que representa el $1.3 \%$ del total. De ellos, $51 \%$ estaban inscritos en programas de educación continua, $36 \%$ en estudios de posgrado y $13 \%$ en estudios de licenciatura (Rama, 2007).

\section{COBERTURA DE LA EDUCACIÓN SUPERIOR EN MÉXICO Y PARTICIPACIÓN DE LA EDUCACIÓN VIRTUAL}

La matrícula de educación superior en México ha experimentado un importante crecimiento en años recientes. Mientras que en el ciclo 2006-2007 la matrícula fue ligeramente superior a los dos y medio millones de estudiantes, para el ciclo actual 2010-2011 se estima que esa cifra rebasa los tres millones. Este crecimiento se observa en las modalidades presenciales y en las no presenciales, si bien la escolarizada representa el 91\% (Turián, 2011).

No obstante, México, aún con estos avances, está por muy por detrás de países como Estados Unidos, Canadá, Argentina, Uruguay y Chile, cuya atención a la demanda de educación superior supera el 60\%. Resulta evidente que deben tomarse 
las medidas que amplíen nuestra capacidad de atención a los miles de jóvenes y personas de otras edades que no tienen acceso a este nivel educativo.

Como se dijo antes, las instituciones de nivel superior en el mundo están aprovechando las ventajas que representa la educación a distancia y especialmente la modalidad en línea para ampliar su matrícula. Además, en un entorno donde los conocimientos se multiplican a velocidades impresionantes, esta modalidad está siendo especialmente útil para ayudar a los miles de egresados de los sistemas educativos de nivel superior a mantenerse al día en sus campos profesionales respectivos.

En el caso particular de México, si las cifras de la Subsecretaría de Educación Superior son correctas, la matrícula de educación superior en programas no escolarizados ronda los 300000 estudiantes (Tuirán, op. cit.). Sin embargo, aún siendo ciertas dichas cifras, no se sabe cuántos de esos estudiantes realizan sus estudios realmente a distancia o lo hacen en la modalidad abierta que es básicamente presencial sabatina. En nuestro país el último estudio sistemático sobre la educación a distancia en México fue el realizado por la Asociación Nacional de Universidades e Instituciones de Educación Superior, ANUIES, y publicado en 2003 (Ortiz, 2003). Es evidente la necesidad para México de conocer con más detalle las particularidades de este subsistema. Sin embargo, podemos afirmar que la modalidad a distancia está en su etapa inicial en nuestro país.

Muestra de esta etapa inicial son las iniciativas del Espacio Común de Educación Superior a Distancia, ECOESAD y del programa de Educación Abierta y a Distancia, ESAD. El ECOESAD es una iniciativa de las instituciones públicas de educación superior mexicanas que se han asociado para ofrecer, colectivamente, la oferta académica de sus miembros. Prácticamente todas las universidades autónomas forman parte de este organismo, sin embargo, no se dispone de información sobre el número de estudiantes participantes. La Secretaría de Educación Pública, SEP, por su parte, lanzó recientemente el ESAD que ofrece 13 carreras universitarias con una matrícula actual de 27234 alumnos y de acuerdo con autoridades de la SEP, esta cifra crecerá a 80 mil para el 2012 (Tuirán, op.cit.).

\section{RETOS DE LA EDUCACIÓN SUPERIOR VIRTUAL EN MÉXICO}

Frente al vigoroso panorama mundial de educación superior virtual uno puede preguntarse por qué México no ha incursionado con más energía en esta modalidad. 
A nuestro parecer habría al menos tres causas principales: 1) acceso a la tecnología y 2) reducida oferta y 3 ) calidad desconocida.

De acuerdo con datos del Instituto Nacional de Geografía y Estadística, INEGI, en 2010, el número de personas con acceso a Internet en México era de sólo 32.8 millones, no obstante, ha crecido $20.6 \%$ respecto del año 2009. De este grupo, la población entre los 12 y los 34 años de edad fue quien hizo mayor uso de Internet, representando el 66.8\% del total. (INEGI, 2010). De acuerdo con el Banco Mundial este panorama se refleja en el hecho de que para 2009 en México había sólo 26.5 personas usuarias de Internet por cada 100, situación que contrasta con la de Colombia con 45.5, Brasil 39.2, Chile 34, Venezuela 31.2, Argentina 30.1 y Perú 27.7; y ni qué decir de Estados Unidos donde 78 personas de cada 100 tiene acceso a Internet, o Canadá con 77.7 (Banco Mundial, 2011).

Otra limitante es la reducida oferta de programas a distancia, y en particular, en la modalidad en línea. Como se dijo antes en México no existe un monitoreo sistemático de la evolución de esta modalidad, habiéndose publicado el último estudio en 2003. El Directorio Nacional de Instituciones de Educación Superior de la Asociación Nacional de Universidades e Instituciones de Educación Superior, ANUIES, refiere 91 instituciones de educación superior que ofrecen programas en la modalidad a distancia (ANUIES, 2011), pero en el directorio no se indica cuántas de ellas emplean Internet como recurso tecnológico principal para llevar a cabo los estudios, y otros procesos académicos y administrativos para considerarse como una institución virtual (Facundo, 2004).

Una búsqueda en Internet realizada en el mes de abril de 2011 por el Instituto Consorcio Clavijero, ICC, en el motor de búsqueda Google y empleando los descriptores: educación en línea en México, universidades virtuales en México, licenciaturas virtuales o en línea en México, oferta académica en línea en México y educación a distancia en México, arrojó como resultado la existencia de 50 instituciones en línea (muchas presenciales y en línea). Al agregar al propio ICC y su oferta educativa, se tienen 51 instituciones con 1071 programas, de los cuales 318 son de licenciatura (29\%), 220 de maestría (20\%), 406 programas de educación continua (37\%) y 127 (11\%) en la categoría de otros. Se observa que la modalidad en línea es empleada mayoritariamente para ofrecer cursos de educación continua, si bien es promisorio saber que en segundo lugar figuran los programas de licenciatura.

Un tercer elemento, que ha podido contribuir a la evolución lenta de la educación superior a distancia en México, es el poco conocimiento que se tiene de su calidad. Si 
bien el país ha avanzado significativamente en el establecimiento de un mecanismo confiable de evaluación y acreditación de la educación superior presencial, mediante los Comités Interinstitucionales de la Evaluación de la Educación Superior, CIEES, y el Consejo para la Acreditación de Programas de Educación Superior, COPAES, la participación de estos organismos es, en el primer caso, reciente, y, en el segundo, inexistente. Los CIEES reportan haber evaluado, para el mes de marzo de 2011, 17 programas académicos virtuales y tener cuatro más en proceso de evaluación (De la Garza, 2011).

En el siguiente apartado se revisan diversos modelos de evaluación de programas virtuales para presentar después el modelo empleado por el ICC para promover y asegurar la calidad de sus servicios.

\section{MODELOS DE ASEGURAMIENTO DE LA CALIDAD DE LA EDUCACIÓN SUPERIOR VIRTUAL}

En este apartado se da cuenta de distintas experiencias internacionales y nacionales de modelos de evaluación de programas a distancia en línea. Se reconoce que es responsabilidad de los gobiernos, de las autoridades educativas, de las asociaciones regionales y nacionales, y de las propias instituciones educativas asegurar la calidad de la formación universitaria en línea. La proliferación de instituciones virtuales y de programas a distancia hace indispensable regular y normar esta modalidad educativa con el fin de asegurar que los estudios realizados sean válidos y de calidad reconocida.

Los modelos propuestos para la evaluación de la calidad de la educación virtual son diversos pero todos coinciden en señalar ciertos componentes como fundamentales para asegurar la calidad de estos estudios. A continuación, se revisan los modelos propuestos por el Council for Higher Education Accreditation, CHEA de los Estados Unidos de América, el Centro Virtual para el Desarrollo de Estándares de Calidad para la Educación a Distancia en América Latina y el Caribe y los CIEES de México.

El modelo propuesto por CHEA para la evaluación y, en su caso, acreditación de los programas de educación superior a distancia es el resultado del acuerdo tomado en 2001 por las ocho organizaciones acreditadoras regionales de ese país (CHEA, 2002). El modelo establece el conjunto de características que deben observarse en el funcionamiento de las instituciones educativas para asegurar la calidad de la educación a distancia. Las siete áreas objeto de análisis en los procesos de evaluación 
son: misión institucional, estructura organizacional, recursos institucionales, curriculum y enseñanza, apoyo al personal docente, apoyo a estudiantes, y resultados del aprendizaje de los estudiantes.

A partir de estos rasgos principales las organizaciones acreditadoras regionales han establecido estándares aplicables a cada uno de ellos de acuerdo con sus marcos de referencia y valores compartidos, pero siempre en consonancia con lo establecido en los siete atributos requeridos para asegurar la calidad de la educación a distancia. El modelo es general pero a la vez lo suficientemente comprehensivo del conjunto de factores que influyen en la calidad de la educación a distancia y permite atender enfoques particulares de las diferentes asociaciones acreditadoras. La manera cómo se expresan los estándares difieren pero no en la atención puesta en alguno de los factores relevantes de esta modalidad.

El modelo del CHEA atiende con suficiencia los aspectos normativos, de planeación, desarrollo, desempeño y resultados de los programas a distancia. El modelo considera la medida en que los programas en esta modalidad contribuyen al logro de los propósitos que persigue la institución, valora la capacidad organizativa y estructural para conducir programas a distancia, resalta la importancia del diseño de los cursos y los materiales de aprendizaje, verifica la habilitación de los alumnos y los docentes y de los apoyos recibidos para el uso de las tecnologías con fines educativos, así como evalúa los resultados de los aprendizajes de los estudiantes.

El modelo propuesto por Centro Virtual para el Desarrollo de Estándares de Calidad para la Educación a Distancia en América Latina y el Caribe surge de la iniciativa del Banco Interamericano de Desarrollo y de la Asociación Iberoamericana de Educación Superior a Distancia, así como de un amplio grupo de especialistas provenientes de instituciones latinoamericanas y españolas. El trabajo realizado por el centro es el resultado de un análisis detallado de las experiencias de educación superior a distancia y de su evaluación en muchos de los países de América Latina y de la Unión Europea.

El enfoque seguido es uno que promueve la mejora continua y divide en dos grandes bloques los aspectos analizados de los programas a distancia. Por una parte, se consideran los elementos facilitadores que incluyen lo siguiente: liderazgo y estilo de gestión, políticas y estrategias, desarrollo de las personas, recursos y alianzas y destinatarios y procesos educativos y, por la otra, los resultados alcanzados por las instituciones en sus programas a distancia expresados en lo siguiente: resultados de los destinatarios y de los procesos educativos, resultados en el desarrollo de las 
personas, resultados sociales y resultados globales. El enfoque tiene importantes aplicaciones prácticas y también sirve de marco de referencia para adaptarle nuevos elementos de análisis para responder a condiciones particulares de las instituciones (Centro Virtual para el Desarrollo de Estándares de Calidad para la Educación Distancia en América Latina y el Caribe, 2003).

La metodología propuesta en México por los CIEES para la evaluación de los programas a distancia incluye 4 ejes, 11 categorías y 80 indicadores (CIEES, 2010). En el eje de intencionalidad se analizan las categorías de normatividad y políticas generales y planeación y evaluación; en el eje de estructura se revisan las categorías de modelo educativo y plan de estudios, alumnos, personal académico y servicios de apoyo estudiantil; en el eje de infraestructura se considera a las instalaciones, equipo y servicios; y, por último, en el eje de resultados se incluyen las categorías de trascendencia del programa, productividad académica, vinculación con los sectores de la sociedad y aplicación de la plataforma tecnológica. De hecho, el modelo es el mismo al empleado para la evaluación de programas presenciales con la adición de la categoría de "aplicación de la plataforma tecnológica". En principio, se podría estar de acuerdo en usar una metodología común para evaluar los programas de cualquier modalidad, puesto que la calidad de los aprendizajes construidos por los estudiantes y de los servicios de apoyo recibidos no depende, necesariamente, de la modalidad. Sin embargo, no deja de llamar la atención la señal que se proyecta al ubicar las propiedades típicas de la modalidad a distancia en la plataforma tecnológica. Por otra parte, la extrapolación que se hace del modelo de evaluación de programas escolarizados a los programas en línea supone como idénticas las condiciones de operación de ambos sistemas, sin serlo. Un ejemplo que ilustra este hecho es el caso de la eficiencia terminal. Mientras en la educación escolarizada es común considerar cohortes generacionales que inician y concluyen sus estudios al mismo tiempo, en las modalidades en línea se promueva la flexibilidad para que el tiempo que le tome al estudiante terminar sus estudios responda a sus condiciones particulares. Es decir, en México es conveniente profundizar aún más en el establecimiento de lo que debe ser considerado como esencial a ser evaluado de los programas en línea. La propuesta metodológica de los CIEES incluye, no obstante, precisiones respecto a variables fundamentales en la educación virtual que servirán, de emplearse con propiedad, para asegurar la calidad de esta modalidad como lo son: la legalidad de los estudios, el modelo educativo, el diseño de las experiencias de aprendizaje y de los materiales educativos, la información y capacitación de estudiantes y profesores, los medios de comunicación entre profesores y alumnos y entre alumnos, los procedimientos de evaluación, la plataforma tecnológica y los sistemas de soporte académico, administrativo y tecnológico requeridos. 
Al comparar los tres modelos de aseguramiento de la calidad se hace evidente la preocupación en ellos, con distintos niveles de especificidad, por resaltar la importancia de evaluar la correspondencia entre la modalidad a distancia y la misión institucional, la capacidad y compromiso institucional en términos organizacionales y presupuestales, el personal académico, la infraestructura tecnológica, los apoyos académicos, administrativos y tecnológicos, la calidad de su oferta en cuanto a pertinencia y respuesta a estándares y, especialmente, conocer los resultados de los aprendizajes de los estudiantes. En síntesis, que los estudios sean válidos y de calidad reconocida externamente.

\section{MODELO DE ASEGURAMIENTO DE LA CALIDAD EN EL INSTITUTO CONSORCIO CLAVIJERO}

A continuación se presenta el modelo que opera el Instituto Consorcio Clavijero, ICC, para promover la calidad de sus programas académicos. Su diseño emplea nociones de modelos de evaluación equivalentes a los revisados en la sección previa, pero propone un modelo de evaluación que responde a las necesidades propias del instituto. Para el ICC el estudiante no es sólo el centro del proceso educativo, sino de toda la operación del instituto. De allí que el modelo de aseguramiento de la calidad considere la totalidad de elementos que influyen en la calidad de los procesos académicos y administrativos de ingreso, permanencia y egreso de los estudiantes. El modelo responde, además, a los factores responsables de las buenas prácticas en la educación a distancia virtual relativas a la interacción del estudiante con materiales de aprendizaje adecuados, la interacción del estudiante con su profesor y con otros estudiantes y que el estudiante reciba el soporte académico, administrativo y tecnológico requerido (Anderson, 2004). Atiende, asimismo, en lo posible, la propuesta metodológica de los CIEES y, por último, promueve los atributos de programas que fomentan el aprendizaje significativo del estudiante de educación superior a distancia propuestos por Chikering y Gamson (2004) y Shieh, Gummer y Niess (2008), entre otros.

Los elementos considerados en el modelo son los siguientes: pertinencia de la oferta educativa, legalidad de los programas, calidad de cursos y materiales, perfil del profesor, acceso a la tecnología, inducción de estudiantes, apoyo estudiantil, desempeño de profesores, aprendizaje, satisfacción de estudiantes, seguimiento de egresados y plataforma tecnológica.

Para cada elemento se describe en seguida la manera cómo se asegura su calidad proporcionando en los casos que corresponda información cuantitativa. 
En particular, se dará cuenta detallada de la información sobre el desempeño de profesores y alumnos y la manera cómo ésta se emplea para retroalimentar los procesos académicos, tecnológicos y administrativos correspondientes.

\section{Pertinencia de la oferta educativa}

El ICC ofrece siete programas de licenciatura y siete programas de técnico superior universitario (TSU) correspondientes, además de una oferta variada de educación continua. Los programas de TSU y licenciatura fueron diseñados a partir de estudios de factibilidad realizados en la Universidad Veracruzana en 2004, siguiendo la metodología empleada por la Coordinación de Universidades Tecnológicas de la Secretaría de Educación Pública. Por tanto, se entrevistó a empresarios, funcionarios públicos, padres de familia, estudiantes de bachillerato y se analizó la información disponible sobre empleo, vocación productiva regional y tendencias productivas en el estado de Veracruz. La evolución de la matrícula en el instituto no ha sido lo homogénea que se esperaba. Existe una concentración de la matrícula en las carreras de Ingeniería en Sistemas con 32\% de la población estudiantil en licenciatura en Gestión de la Educación y Capacitación con 15\%.

La razón de esta situación es, sin duda, multicausal en la que intervienen factores relacionados con preferencias culturales, así como una difusión probablemente insuficiente de la oferta restante. En próximas fechas se llevará a cabo la revisión cabal de la oferta del instituto con el fin de impulsar su mayor pertinencia. En cualquier caso, lo relevante aquí es destacar la necesidad de que los programas en línea se sustenten en los resultados de análisis sistemáticos que determinen si la oferta propuesta por la institución responde a las necesidades de los estudiantes y a las de su entorno económico y social.

\section{Legalidad de los programas académicos}

El ICC se creó originalmente como una asociación civil integrada por universidades públicas y privadas, por lo que sus programas obtuvieron, en su momento, el reconocimiento de validez oficial de estudios a partir de un decreto secretarial en el que se establecieron las condiciones y requisitos que debían cumplir las instituciones que ofrecieran, en Veracruz, programas en modalidades no escolarizadas, a distancia y virtuales. Sin embargo, en febrero de 2010, la personalidad jurídica del ICC cambió a organismo público descentralizado, por lo que ahora, como institución pública de educación superior, sus programas deben 
ser registrados en la Dirección General de Profesiones de la SEP, proceso que ha sido concluido satisfactoriamente. La legalidad de los estudios ofrecidos en las modalidades a distancia, pero especialmentelas virtuales, es motivo de preocupación legítima por las autoridades educativas y por la sociedad en general.

La oferta de programas de licenciatura y posgrado es abundante pero mucha de ella es de calidad dudosa. Los estudiantes de esta modalidad deben tener la certeza de que sus estudios serán reconocidos como válidos en el país y en el extranjero. La legalidad de los programas es entonces una condición fundamental de aseguramiento de la calidad.

\section{Calidad de los cursos y de los materiales de aprendizaje}

Los cursos que integran los programas académicos del ICC se han desarrollado con la participación de equipos interdisciplinarios integrados por expertos en contenido, diseñadores instruccionales, diseñadores gráficos, expertos en multimedia, programadores, revisores de estilo y evaluadores. Los cursos se han diseñado con base en un enfoque de competencias y todos siguen una estructura común; así mismo, cumplen con estándares de diseño instruccional, diseño de la interfaz y de publicaciones multimedia (Mercado, 2007; Fahy, 2004). El modelo educativo que sirve de fundamento al diseño de los cursos y los materiales de estudio considera al estudiante como eje principal del proceso de enseñanza-aprendizaje. Los aportes de la psicología educativa y, en particular, los trabajos de la corriente constructivista han sido fundamentales como soporte teórico. El modelo reconoce que se aprende haciendo, por lo que todas las actividades de aprendizaje diseñadas conducen a la realización de un producto que sirve de testimonio de su aprendizaje y es empleado por el profesor para calificar su desempeño. En cada ciclo se recogen opiniones de estudiantes y profesores que sirven para realizar ajustes y mejoras a los contenidos y dinámicas de trabajo. Además, la información sobre el desempeño de los alumnos sirve para identificar cursos cuyas tasas de calificación sean bajas lo que puede indicar la necesidad de revisión y/o rediseño de los cursos o materiales, en su caso. Más adelante se ilustrará un ejemplo de este análisis.

\section{Perfil del profesor}

Un factor decisivo en la calidad de los programas virtuales es el papel que desempeñan los profesores. La separación física y temporal entre estudiantes y alumnos obliga a disponer de un sistema eficiente de atención e interacción con el 
estudiante. En el ICC cada profesor atiende a un máximo de 35 estudiantes por curso y sólo pueden tener a su cargo un máximo de tres cursos. Los profesores responsables de la conducción de los cursos son seleccionados mediante una convocatoria pública. En la convocatoria se describen las responsabilidades y condiciones de la participación como docente en el ICC. La selección de los maestros privilegia el disponer de estudios de posgrado y experiencia docente en la modalidad virtual. No obstante, todos los profesores deben realizar un curso introductorio a la modalidad virtual para familiarizarse con los contenidos y la plataforma tecnológica. El profesor es contratado por ciclo y remunerado con base en dos esquemas: un pago fijo más un bono de desempeño establecido a partir del cumplimiento de diez criterios de evaluación. Más adelante se proporciona un análisis de estos criterios y se demuestra su cumplimiento.

\section{Acceso a la tecnología}

Ya se mencionó antes que una barrera que frena el desarrollo de la modalidad virtual en México es la capacidad tecnológica insuficiente para el acceso de la población al uso de computadoras y de Internet. El ICC, en respuesta a esta limitante, ha desarrollado una red de 239 Aulas Clavijero distribuidas en zonas urbanas y rurales del estado de Veracruz. Las aulas cuentan con equipos de cómputo, acceso gratuito a Internet y disponen de un operador responsable de mantener el buen funcionamiento de los equipos y brindar apoyo técnico a los usuarios. Los alumnos que carecen de equipos de cómputo o de acceso adecuado a Internet pueden acudir a las aulas y emplear gratuitamente las instalaciones y servicios disponibles. La mayoría de las aulas se ubica en planteles educativos del nivel de media superior y bibliotecas públicas lo que ayuda a difundir las oportunidades educativas que representa el uso de las tecnologías de la información y la comunicación.

\section{Inducción de estudiantes}

El proceso de ingreso a los programas del ICC se realiza en línea. Este proceso se inicia mediante la información proporcionada en el portal del instituto (www. clavijero.edu.mx) que describe al estudiante prospecto todo lo que debe hacer para inscribirse. La información busca ser lo más clara posible y se acompaña en ocasiones de videos que refuerzan la información escrita. Desde el inicio del proceso, el estudiante recibe el apoyo del personal adscrito al Centro de Apoyo Estudiantil, CAE, quienes establecen contacto con el aspirante mediante chat, correo electrónico y teléfono para aclarar dudas posibles. El personal del CAE acompaña al estudiante 
en los pasos siguientes. Una vez que el estudiante ha llevado a cabo las instrucciones para su ingreso y realizado los pagos correspondientes, debe tomar un curso de inducción para conocer la plataforma tecnológica empleada en el ICC y diferentes estrategias que deberá usar para organizar su lugar y tiempo de estudio y distribuir su carga de trabajo durante la semana.

\section{Apoyo estudiantil}

Una vez inscrito el estudiante se le asigna un consejero integrante del CAE, quien lleva el seguimiento del trabajo del estudiante durante sus estudios. La plataforma educativa empleada en el ICC permite identificar si el estudiante está activo y cumpliendo a tiempo con sus tareas y trabajos o se está retrasando en sus actividades. En ambos casos el consejero interviene por chat, correo electrónico o por teléfono para reconocer el esfuerzo y cumplimiento de las actividades o para motivar a los estudiantes a retomar su trabajo y evitar rezagarse en la entrega de sus compromisos. El consejero cuenta con una ficha de cada uno de los estudiantes asignados en la que dispone de información personal y académica de los estudiantes a su cargo. Asimismo, debe llevar un registro de todos los contactos realizados y la situación particular de cada caso.

\section{Desempeño de profesores}

Como se dijo antes, en los sistemas de educación a distancia, y en particular en los programas en línea, el trabajo desarrollado por los profesores es fundamental.

En todos los modelos de aseguramiento de calidad revisados la figura del docente ocupa un lugar destacado. Y no podría ser de otra manera si consideramos que el estudiante está físicay temporalmente distante. En el ICC se asegura quelos profesores estén habilitados en las herramientas de comunicación disponibles en la plataforma tecnológica empleada para el desarrollo de los cursos y se aprovecha la propia plataforma para llevar a cabo el seguimiento de su trabajo. Para hacer transparente las expectativas respecto al desempeño que deben demostrar los profesores, en el ICC se estipula, desde el mismo contrato de servicios profesionales, el conjunto de criterios que deber ser atendidos. Los profesores conocen de antemano que serán evaluados conforme a su cumplimiento y que su remuneración se determinará, en parte, con base en ello.

En el Instituto Consorcio Clavijero se trabaja en el sistema de competencias laborales no sólo en la producción de cursos, sino también en su implementación, 
por ello los docentes reciban una retribución con base en su desempeño, por lo que fueron establecidos los criterios que se detallan en la tabla 1. Para cada uno de ellos se establecieron indicadores de resultados que muestran el grado de su cumplimiento y el nivel de atención proporcionado por el facilitador en la impartición de los cursos en línea.

\begin{tabular}{|c|c|c|}
\hline & Criterio & Indicador \\
\hline 1. & Mensaje de bienvenida & $\begin{array}{l}\text { Mensaje de bienvenida enviado al inicio del } \\
\text { ciclo escolar. }\end{array}$ \\
\hline 2. & Mensaje semanal & $\begin{array}{l}\text { Envío de mensaje por semana con las } \\
\text { indicaciones de trabajo de la semana } \\
\text { correspondiente. }\end{array}$ \\
\hline 3. & Mensaje de despedida & $\begin{array}{l}\text { Mensaje de despedida enviado el lunes } \\
\text { posterior al fin de curso. }\end{array}$ \\
\hline 4. & Chat semanal & $\begin{array}{l}\text { Organización de, por lo menos, una hora } \\
\text { semanal de chat para solución de dudas } \\
\text { e interacción con el estudiante en tiempo } \\
\text { real. }\end{array}$ \\
\hline 5. & $\begin{array}{l}\text { Retroalimentación a dudas y } \\
\text { comentarios del estudiante }\end{array}$ & $\begin{array}{l}\text { Respuesta a mensajes y dudas del } \\
\text { estudiante antes de } 24 \text { horas hábiles. }\end{array}$ \\
\hline 6. & Calificación de tareas & $\begin{array}{l}\text { Calificación y retroalimentación de tareas } \\
\text { del estudiante antes de } 48 \text { horas hábiles. }\end{array}$ \\
\hline 7. & $\begin{array}{l}\text { Interacción continua con los } \\
\text { estudiantes }\end{array}$ & $\begin{array}{l}\text { El docente no se ausenta de la plataforma } \\
\text { por más de } 24 \text { horas hábiles, de lunes a } \\
\text { sábado. }\end{array}$ \\
\hline 8. & $\begin{array}{l}\text { Evaluación de los estudiantes } \\
\text { respecto de la calidad de } \\
\text { atención y desempeño del } \\
\text { facilitador }\end{array}$ & $\begin{array}{l}\text { Promedio de evaluación en la encuesta de } \\
\text { opinión del estudiante, mayor a } 3 \text {. }\end{array}$ \\
\hline 9. & $\begin{array}{l}\text { Entrega oportuna de actas de } \\
\text { calificaciones }\end{array}$ & $\begin{array}{l}\text { Recepción oportuna de actas de } \\
\text { calificaciones electrónicas. }\end{array}$ \\
\hline & Rango de aprobados & $\begin{array}{l}67 \text { al } 100 \% \text { de los estudiantes inscritos en } \\
\text { el curso, que aprueban. }\end{array}$ \\
\hline
\end{tabular}

Tabla 1. Criterios de desempeño del facilitador en línea del Instituto Consorcio Clavijero 
Estos criterios, comunes a cada programa académico, garantizan al alumno una atención suficiente por parte del facilitador y se materializan en la plataforma educativa Eminus empleada por la institución de la siguiente forma (ver tabla 2):

\begin{tabular}{|c|c|c|c|c|c|c|c|c|c|c|c|c|c|}
\hline \multicolumn{14}{|l|}{ 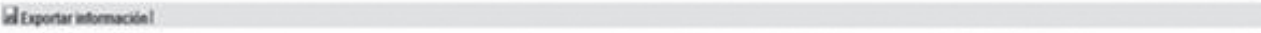 } \\
\hline \multirow{2}{*}{ 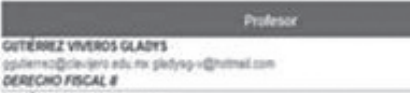 } & \multicolumn{4}{|c|}{ 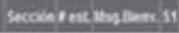 } & \multicolumn{5}{|c|}{ 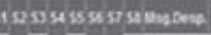 } & \multirow{2}{*}{ 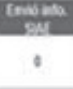 } & \multirow{2}{*}{ Ponas } & \multicolumn{2}{|c|}{ 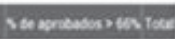 } \\
\hline & 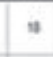 & 4 & 1 & 45 & 21 & 45 & 18 & 2 & . & & & 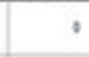 & * \\
\hline 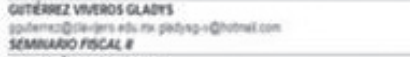 & $x$ & 1 & 1 & if & 18 & 58 & 2. & 21 & 4 & , & ' & 1 & 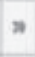 \\
\hline 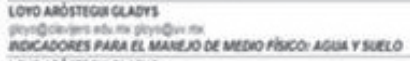 & 107 & 1 & 1 & 212 & 222 & 45 & 8 & 8 & 4 & 4 & 1 & $t$ & $n$ \\
\hline 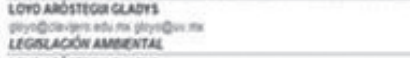 & $n$ & 1 & 1 & 12 & $2 / 2$ & 12 & 1 & 9 & 4 & a & 1 & 4 & H \\
\hline 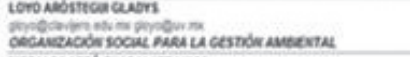 & a & $n$ & 1 & $2 \mid 2$ & $2: 1$ & $1 \mid 2$ & & t & 4 & 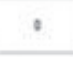 & 1 & 0 & $\boldsymbol{a}$ \\
\hline 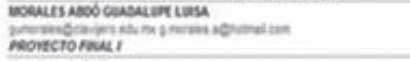 & $w$ & 4 & 1 & 212 & 21 & $4: 2$ & 2 & 1 & 0 & 0 & 1 & 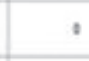 & $n$ \\
\hline 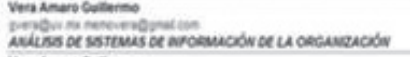 & 10 & 7 & 1 & it 1 & 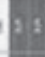 & $1 / 2$ & (1) & 2) & 1 & 0 & 1 & 1 & * \\
\hline 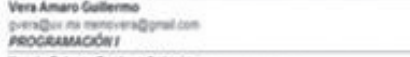 & $\mathrm{k}$ & $x$ & I & 11 & 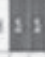 & 12 & (2) & 2 & 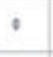 & 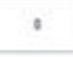 & 1 & 0 & n \\
\hline 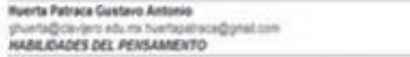 & $g$ & $x$ & 1 & $: 2$ & $2: 2$ & $2: 2$ & 1 & 2 & 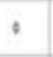 & 0 & 1 & 1 & 4 \\
\hline 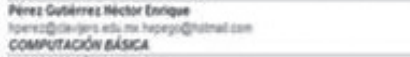 & $\omega$ & 20 & 1 & 14 & 312 & 5 & (5) & 5 & 4 & 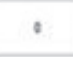 & , & $\circ$ & 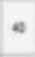 \\
\hline 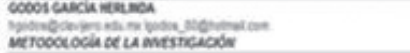 & $w$ & 1 & 1 & 11 & 18 & 41 & 18 & i. & i & 0 & , & , & $\bullet$ \\
\hline
\end{tabular}

Tabla 2. Vista general de la pantalla de desempeño del profesor en línea extraída de la plataforma tecnológica Eminus

A continuación, se presenta el monitoreo semanal que permite asegurar el mínimo de interacción exigida por semana al profesor en línea:

Criterios a cumplir por semana

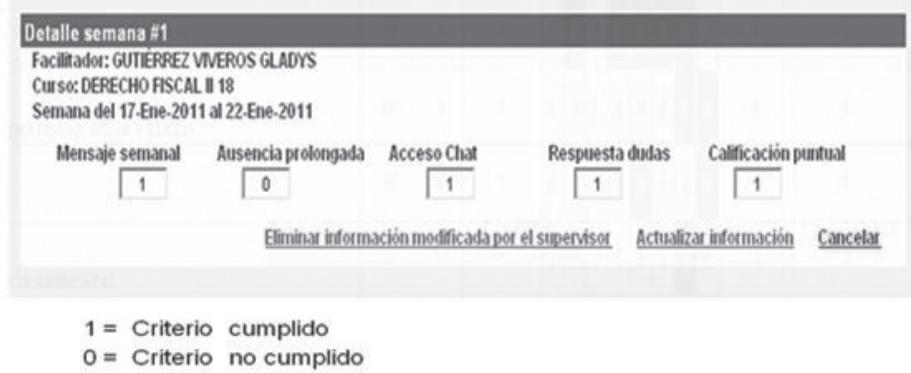

Tabla 3. Vista en detalle semanal de la pantalla del desempeño del profesor en la plataforma tecnológica Eminus 
Esta tabla de desempeño permite dar seguimiento y garantizar la interacción oportuna entre estudiantes y docentes a medida que inicia, avanza y finaliza el proceso educativo, así como identificar el grado de consecución del criterio correspondiente con el objeto de asegurar que el facilitador esté cumpliendo con su labor obligatoria: asesorar al alumno.

Como muestra la figura 1, el $96 \%$ del profesorado en línea del ICC cubre de siete a diez criterios de desempeño y $60 \%$ atiende de nueve a diez, lo que refleja que una mayoría de los docentes contesta oportunamente las dudas planteadas por los estudiantes, corrige oportuna y eficientemente las tareas, retroalimentándolas cualitativamente, provee espacios para la interacción sincrónica y asincrónica e interactúa, de manera formal y estructurada, desde el inicio y hasta el final del proceso educativo. Datos que permiten afirmar que el facilitador en línea del ICC desarrolla su capacidad empática, elemento nodal en la interacción estudiante-profesor, lo cual contribuye a que los alumnos sean cada vez más capaces de autogestionar su aprendizaje. (Ander-Egg, 1999).

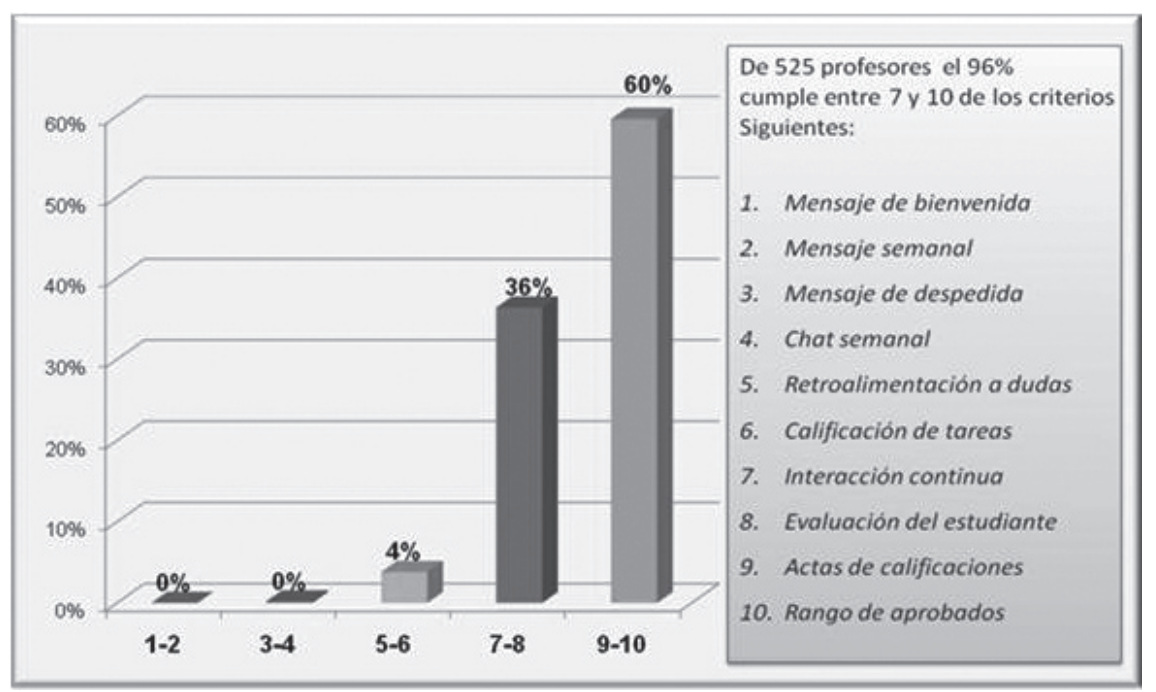

Figura 1. Porcentaje de criterios de desempeño cubiertos por profesores durante los 4 ciclos del año 2010 


\section{Aprendizaje}

Antes de referirnos al aprendizaje de los estudiantes se presenta a continuación información sobre la matrícula y la permanencia del alumnado del ICC. La figura 2 muestra la matrícula por año registrada en el instituto. Puede observarse que el número de estudiantes ha ido creciendo a un ritmo relativamente sostenido. El porcentaje de crecimiento en los cinco años considerados es cercano al $300 \%$.

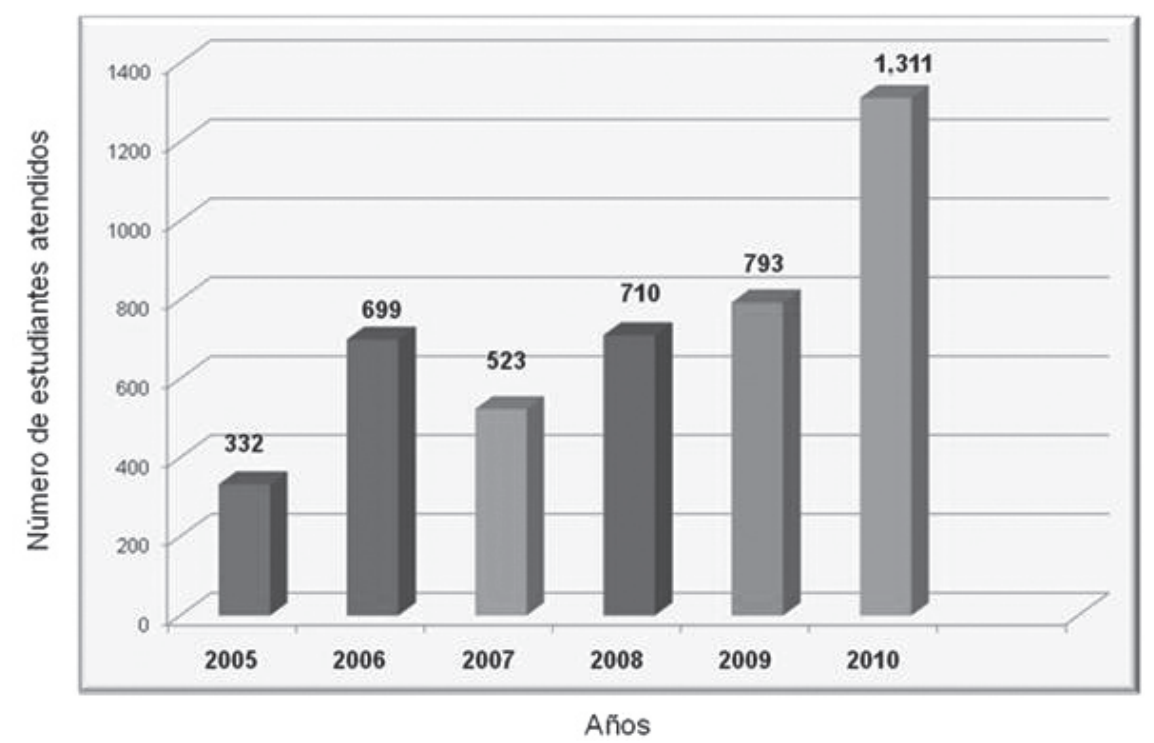

Figura 2. Matricula total del ICC en programas de licenciaturas y TSU, 2005- 2010

Una preocupación legítima en la educación a distancia, y especialmente en su forma en línea, es la deserción estudiantil. No es extraño saber de índices de deserción notablemente superiores a los índices de deserción de los programas presenciales (Díaz, 2002). En el ICC se observa que en el primer año de operaciones la deserción fue alta pero, a partir del segundo año, dicho índice se redujo considerablemente como se muestra en la figura 3 , en donde se aprecia que la deserción bajó de $46 \%$ en 2006 a 29\% en 2010, como resultado, entre otras razones posibles, de un trabajo de seguimiento más eficaz de los estudiantes. 


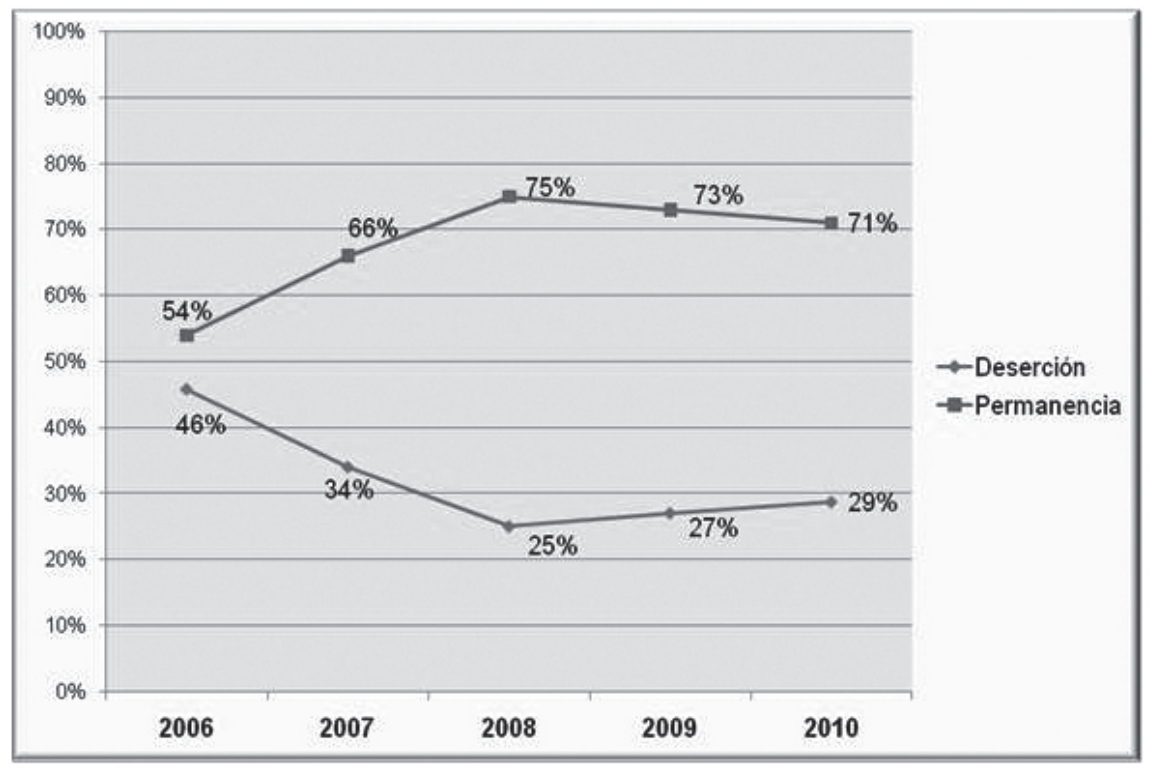

Años

Figura 3. Serie histórica de las tasas de permanencia y deserción de los estudiantes del ICC, $2006-2010$

La fuente primaria de información sobre el aprendizaje de los estudiantes es la evidencia acumulada por los profesores del desempeño de los estudiantes a lo largo de los cursos. En el ICC los cursos, como ya se dijo, están diseñados por competencias que deben ser demostradas por los estudiantes mediante los productos que generan como resultado de las actividades de aprendizaje que emprenden. Los trabajos realizados por los estudiantes son resguardados electrónicamente y sirven de referente en caso de revisiones de estudio o de otro análisis requerido. El profesor otorga al estudiante una nota al finalizar el curso que es la suma de las puntuaciones recibidas en el desarrollo de las actividades de aprendizaje programadas. El estudiante es informado previamente del valor de cada actividad. El ICC emplea la información de las calificaciones del estudiante para determinar la posible existencia de dificultades en algún curso en particular.

La tabla 4 es un ejemplo de este tipo de análisis. Se presenta información respecto de los 16 cursos que reportaron las menores tasas de calificación en el ciclo enero- marzo de 2011. Es evidente la necesidad de identificar las causas responsables de la baja calificación observada en las materias de Inglés y Taller de Lectura y 
Redacción, y tomar las acciones correctivas pertinentes. De hecho, los 16 cursos están considerados como los primeros que serán rediseñados nuevamente.

\begin{tabular}{|c|c|c|c|c|c|c|c|c|}
\hline No. & Curso & 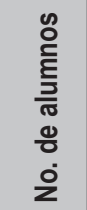 & 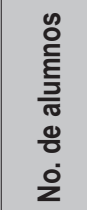 & 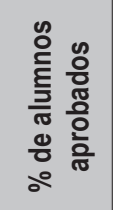 & 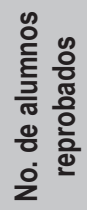 & 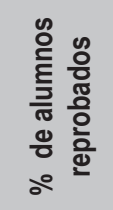 & 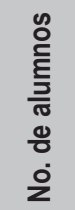 & 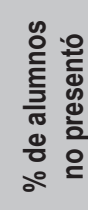 \\
\hline 1 & INGLÉS 1 & 309 & 112 & 36.2 & 172 & 55.7 & 25 & 81.1 \\
\hline 2 & TALLER DE LECTURA Y REDACCIÓN & 292 & 124 & 42.5 & 131 & 44.9 & 37 & 12.7 \\
\hline 3 & FORMULACIÓN DE PROYECTOS & 273 & 117 & 42.9 & 139 & 50.9 & 17 & 6.2 \\
\hline 4 & FUNDAMENTOS DE ADMINISTRACIÓN & 270 & 128 & 47.4 & 133 & 49.3 & 9 & 3.3 \\
\hline 5 & HABILIDADES DE PENSAMIENTO & 267 & 141 & 52.8 & 97 & 36.3 & 29 & 10.9 \\
\hline 6 & $\begin{array}{l}\text { INTRODUCCIÓN A LA EDUCACIÓN A } \\
\text { DISTANCIA }\end{array}$ & 260 & 140 & 53.8 & 103 & 39.6 & 17 & 6.5 \\
\hline 7 & $\begin{array}{l}\text { COMPETENCIAS INFORMATIVAS PARA } \\
\text { EL APRENDIZAJE }\end{array}$ & 243 & 111 & 45.7 & 121 & 49.8 & 11 & 4.5 \\
\hline 8 & COMPUTACIÓN BÁSICA & 236 & 131 & 55.5 & 90 & 38.1 & 15 & 6.4 \\
\hline 9 & ÉTICA CONTEMPORÁNEA & 208 & 135 & 64.9 & 68 & 32.7 & 5 & 2.4 \\
\hline 10 & INGLÉS II & 99 & 64 & 64.6 & 35 & 35.4 & 0 & 0 \\
\hline 11 & PROGRAMACIÓN I & 93 & 43 & 46.2 & 49 & 52.7 & 1 & 1.1 \\
\hline 12 & CÁLCULO PROPOSICIONAL & 83 & 33 & 39.8 & 47 & 56.6 & 3 & 3.6 \\
\hline 13 & MATEMÁTICAS DISCRETAS & 62 & 14 & 22.6 & 37 & 59.7 & 11 & 17.7 \\
\hline 14 & CONTEXTO EMPRESARIAL & 53 & 33 & 62.3 & 20 & 37.7 & 0 & 0 \\
\hline 15 & $\begin{array}{l}\text { FUNDAMENTOS DE CONTABILIDAD Y } \\
\text { FINANZAS }\end{array}$ & 51 & 35 & 68.6 & 16 & 31.4 & 0 & 0 \\
\hline \multirow[t]{2}{*}{16} & $\begin{array}{l}\text { POLÍTICAS Y LEGISLACIÓN } \\
\text { EDUCATIVA }\end{array}$ & 50 & 34 & 68 & 16 & 32 & 0 & 0 \\
\hline & Total & 2849 & 1395 & $48.96 \%$ & 1274 & $44.72 \%$ & 180 & $6.32 \%$ \\
\hline
\end{tabular}

Tabla 4. Relación de cursos con los Índices menores de calificación en el ciclo enero - marzo 2011

\section{Satisfacción de estudiantes}

La satisfacción de los estudiantes es una de las medidas de uso generalizado en los modelos de aseguramiento de calidad de la educación presencial y a distancia (Palmer y Holt, 2008; Brennan y Williams, 2009; Bekele, 2010). Conocer la satisfacción de los estudiantes respecto a los programas educativos, sus maestros y los servicios que reciben es de espacial importancia en la educación en línea por la relación existente entre su nivel de satisfacción y su permanencia como estudiantes (Bollinger y Martindale, 2004; Gaskell, 2009). 
En el ICC se emplean dos instrumentos de evaluación de la opinión de los estudiantes. Uno de ellos se aplica al inicio de la segunda semana de cada curso con el fin de identificar si algún estudiante está teniendo dificultades en su cuenta de correo, para acceder a los materiales de estudio o para contactar a su profesor.

La idea es solucionar cualquier problema al inicio del curso que pueda impedir al estudiante participar oportunamente. El segundo instrumento tiene como uno de sus referentes el modelo de interacción propuesto por Ally (2004) por lo que contiene preguntas sobre la interacción del estudiante con los contenidos, el profesor y la tecnología. Este instrumento se aplica en la octava semana de los cursos y permite conocer la opinión de los estudiantes respecto de la calidad de los cursos y materiales de estudio, del desempeño del profesor y del consejero, la plataforma tecnológica, así como respecto a su propio desempeño como estudiante. El índice de confiabilidad del cuestionario de satisfacción mediante el método de dos mitades y aplicando la fórmula de Spearman- Brown es 0.93 .

Este segundo instrumento ha sido aplicado sistemáticamente durante los últimos cuatro años como podrá observarse en los cuadros siguientes. La figura 4 presenta información agregada histórica de un total de 5201 estudiantes. La evaluación promedio de los estudiantes respecto del curso, profesor, consejero y su autoevaluación fue de 3.4 en 2007 y de 3.5 sobre 4 en los años siguientes.

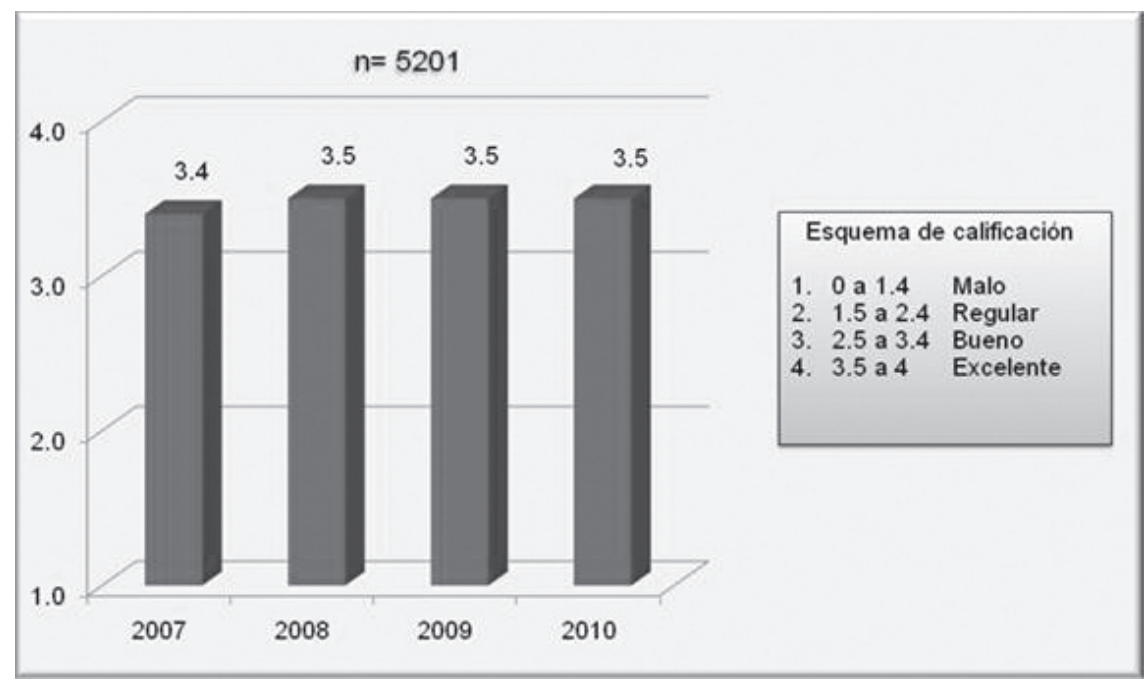

Figura 4. Índice global histórico de satisfacción de estudiantes del ICC de 2007 a 2010 
La figura 5 muestra la satisfacción histórica promedio del mismo número de estudiantes sobre la calidad del curso. En el margen derecho de todos los cuadros se presentan las preguntas que contiene esa sección del instrumento. Aquí, en cuanto al curso, observamos que la opinión de los estudiantes es entre 3.6 y 3.7 sobre 4 para los cuatro años.

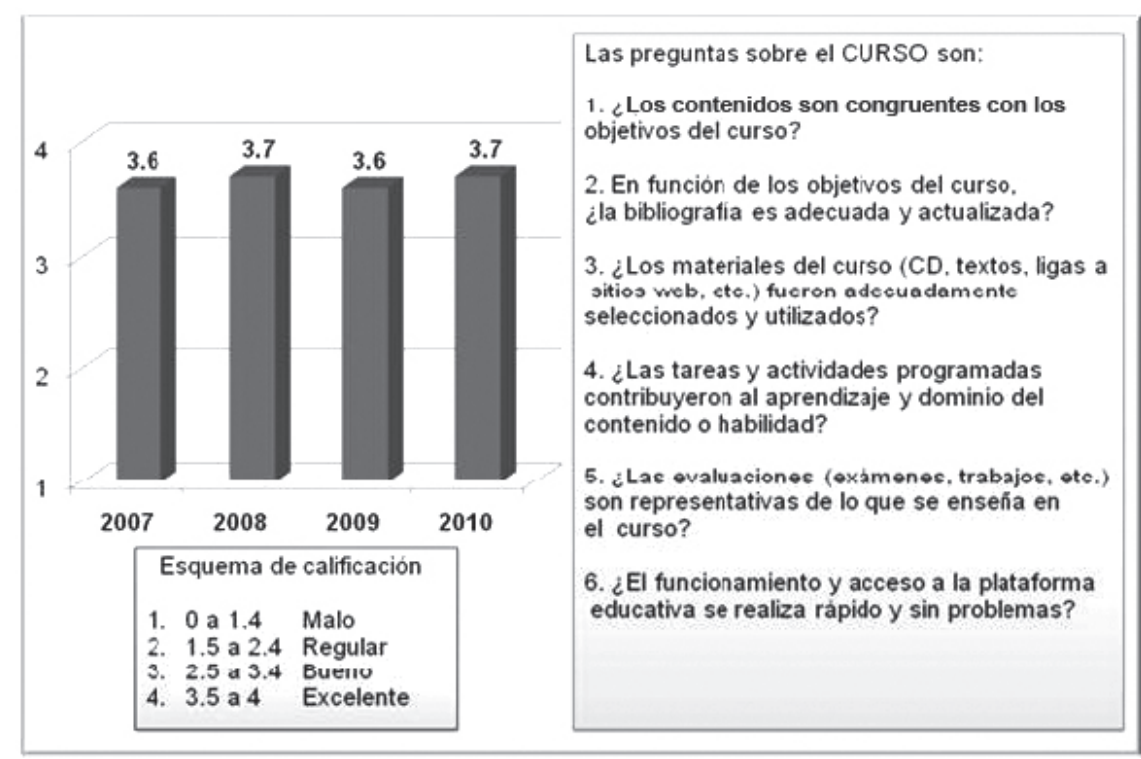

Figura 5. Índice histórico de satisfacción de estudiantes del ICC sobre el curso de 2007 a 2010

La figura 6 presenta la opinión de los estudiantes respecto a su profesor donde también observamos una apreciación favorable de su desempeño con valores de 3.6 y 3.7 sobre una escala de 4 . 


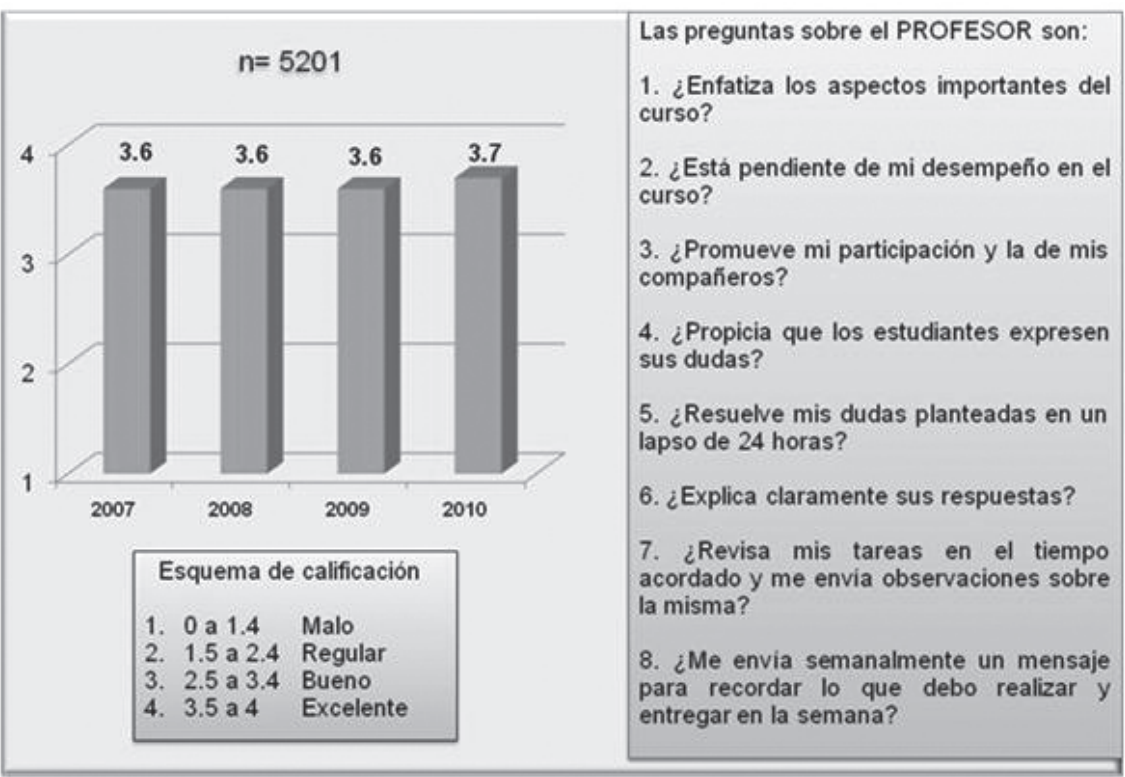

Figura 6. Índice histórico de satisfacción de estudiantes del ICC sobre el profesor de 2007 a 2010

La opinión de los estudiantes sobre su consejero se muestra en la figura 7. Aquí se aprecia una mejora gradual en la percepción de los estudiantes respecto de su consejero pasando de 3.3 a 3.5, pero indicando que aún se tiene un espacio de oportunidad para mejorar la calidad de este servicio.

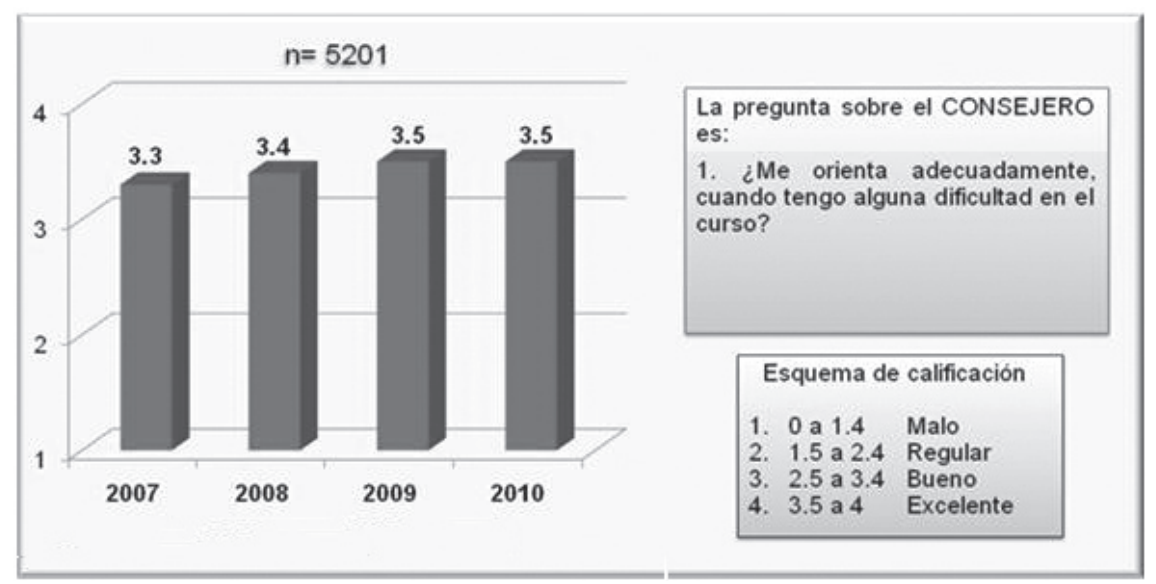

Figura 7. Índice histórico de satisfacción de estudiantes del ICC sobre el consejero de 2007 a 2010 
La opinión de los estudiantes sobre su propio compromiso y dedicación al estudio a distancia se aprecia en la figura 8. Los valores reportados de 3.3 para todos los años indican que están conscientes, hasta cierto punto, de su responsabilidad para participar en esta modalidad y en estos casos que aún podrían haber hecho mayores esfuerzos para mantenerse al corriente en la entrega de sus trabajos y de participar en los espacios de colaboración correspondientes.

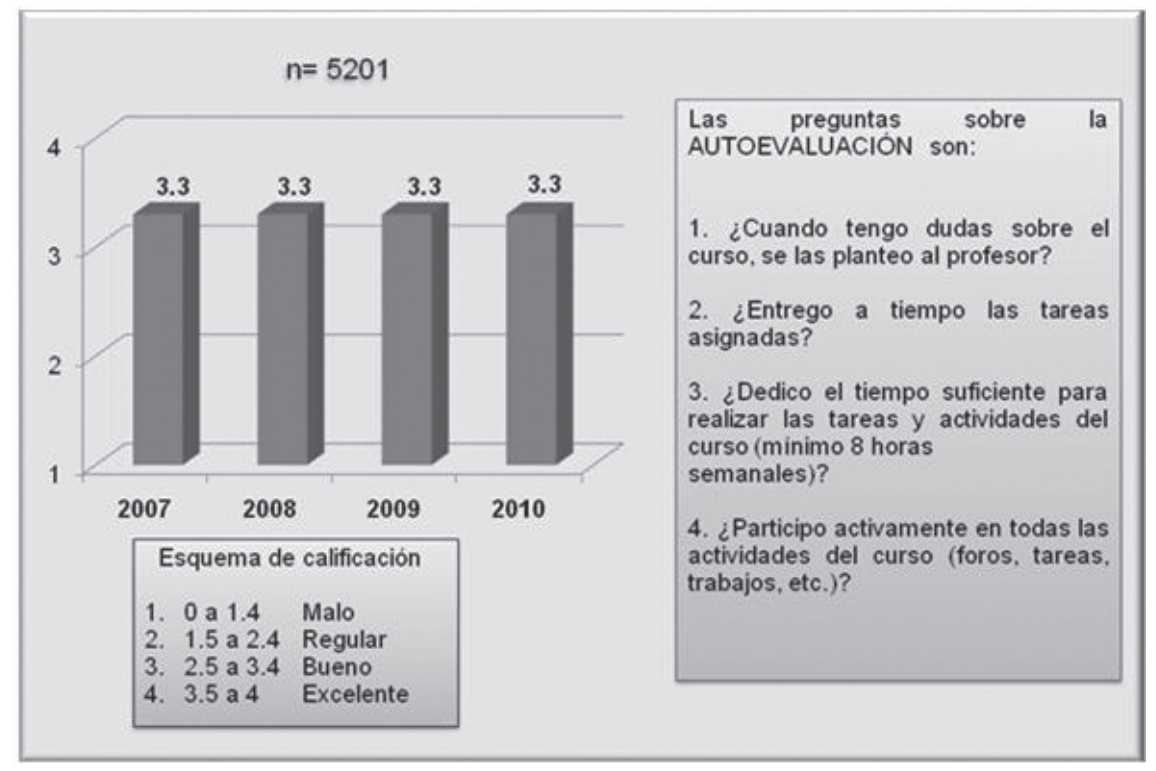

Figura 8. Índice histórico de la autoevaluación de los estudiantes del ICC de 2007 a 2010

La información recogida mediante el cuestionario de satisfacción puede agregarse y desagregarse en diferentes niveles. La figura 9 ilustra la opinión promedio de los estudiantes en las cuatro secciones del instrumento en los siete programas de licenciatura y en dos cursos de educación continua dirigidos a profesores de educación básica del estado de Veracruz y realizados en el ciclo agosto-diciembre de 2010. El total de alumnos incluye a estudiantes de licenciatura y de los cursos de educación continua. Puede apreciarse que la evaluación promedio de la totalidad de programas ofrecidos por el instituto es favorable. 


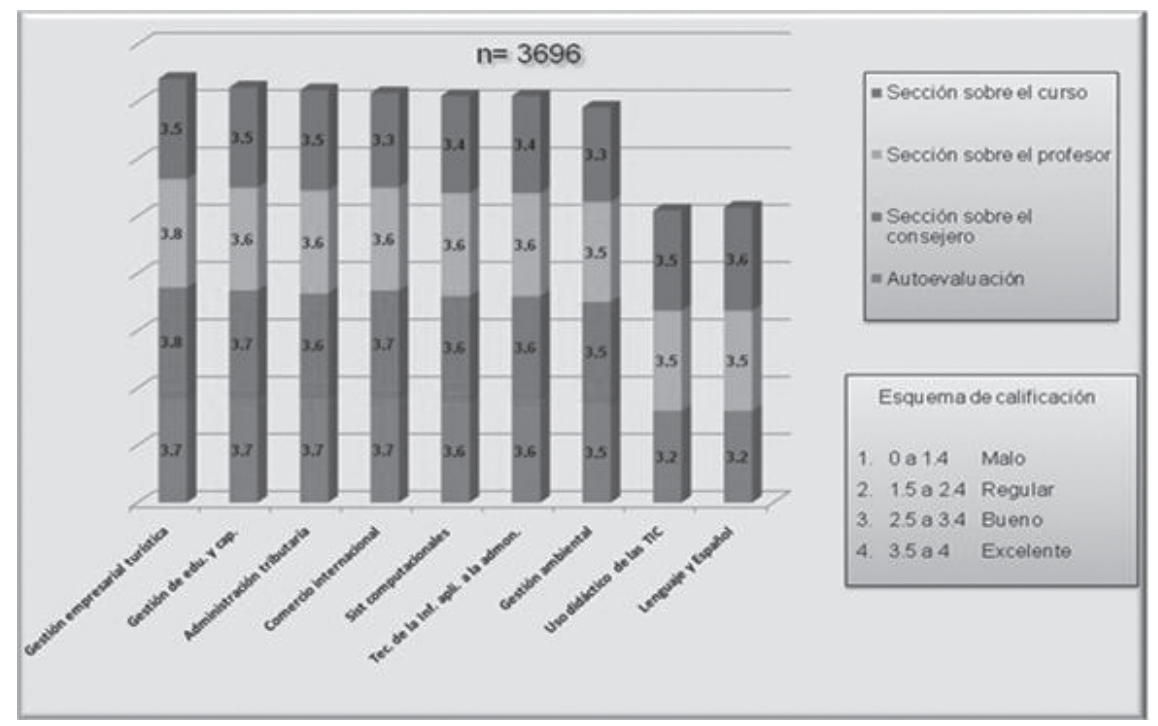

Figura 9. Índice de satisfacción de los estudiantes del ICC en el ciclo agosto - diciembre 2010 de los 7 programas de licenciatura y los 2 cursos de educación continua. Se presenta la información sobre el curso, profesor, consejero y autoevaluación. La información del consejero no se presenta en los programas de educación continua

La opinión de los estudiantes también se conoce por curso y sección. La figura 10 da cuenta de la percepción de los estudiantes respecto de las materias ofrecidas en el ciclo mayo-julio de 2010 en la carrera de Tecnologías de la Información Aplicadas a la Administración, donde la opinión en las cuatro partes del cuestionario oscila entre 3.5 y 4. Por último, la figura 11 ilustra la opinión de los alumnos de tres grupos o secciones diferentes conducidos por tres diferentes docentes en la materia de Ética Contemporánea. En general, los estudiantes perciben una calidad homogénea entre los distintos maestros y grupos de estudio. 
R. MERCADO; M. LóPEZ; G. BALDERAS

El Aseguramiento De La Calidad en el Instituto Consorcio Clavijero

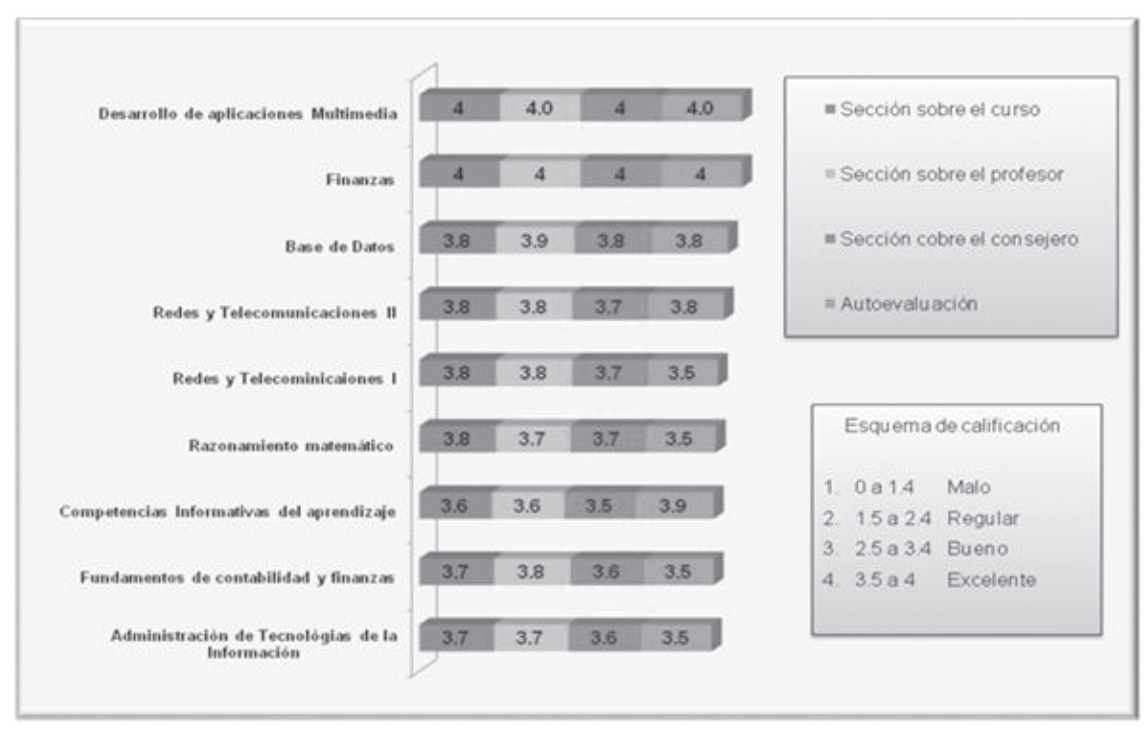

Figura 10. Índice de satisfacción por materia de los estudiantes del ICC de la licenciatura en tecnología de la información aplicada a la administración, ciclo mayo - julio 2010. Se presenta información sobre curso, profesor, consejero y autoevaluación

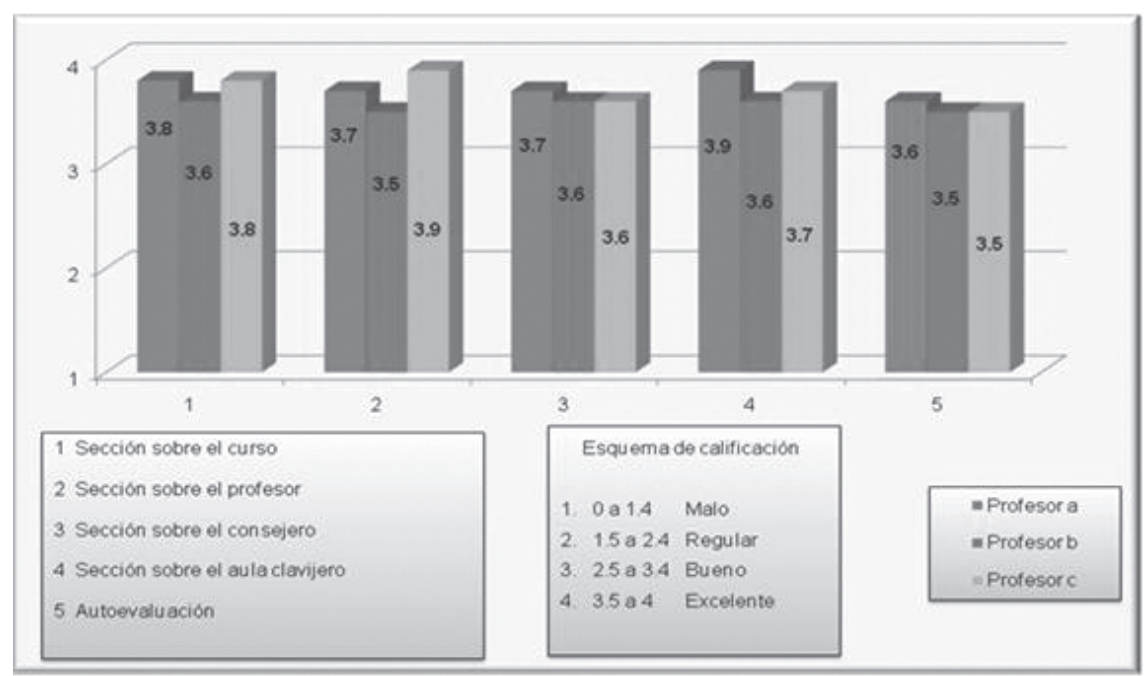

Figura 11. Índice de satisfacción de 3 grupos de la materia de Ética Contemporánea, ciclo octubre - diciembre 2010 
La información obtenida por medio de los cuestionarios de satisfacción es especialmente importante en un modelo de aseguramiento de la calidad de los programas a distancia. Por definición, los estudiantes de esta modalidad no están presentes físicamente en la institución para expresar sus opiniones, sugerencias o quejas. De allí que además del seguimiento continuo que se hace de su desempeño y de la recepción de opiniones enviadas al CAE, los cuestionarios de satisfacción sean una herramienta valiosa para tomar el pulso de la opinión de los estudiantes respecto de los servicios que ofrece el instituto. La información así proporcionada tiene varias aplicaciones pues permite identificar la necesidad de realizar mejoras en los cursos, en la infraestructura tecnológica y en el desempeño de los profesores y consejeros. En el caso del desempeño de los profesores la información de los estudiantes es tomada en cuenta para determinar, parcialmente, el monto final de su remuneración, como para los efectos de la renovación de las contrataciones.

\section{Seguimiento de egresados}

El fin último de los programas académicos del ICC, como el de toda institución de educación superior, es que sus egresados encuentren un trabajo bien remunerado y apropiado a su formación que les permita realizar sus aspiraciones personales y contribuir a la satisfacción de necesidades sociales. En México existe una tradición en el desarrollo de estudios de egresados y prácticamente todas las instituciones públicas y muchas de las privadas realizan esta actividad para la modalidad escolarizada. En el ICC este proceso recién tuvo su inicio a finales del 2010 cuando se graduó el primer grupo de egresados.

En la actualidad los estudiantes universitarios lo son para toda la vida. Un profesionista ya no puede pensar que al concluir sus estudios universitarios termina su proceso formativo. La velocidad con la que se produce nueva información y conocimientos les obliga a mantenerse al día en su campo profesional y laboral. De allí que los estudios de egresados y el contacto frecuente que se mantenga con ellos, representa una fuente de información valiosa para atender las necesidades dinámicas de los egresados en materia de su formación continuada.

\section{Plataforma tecnológica}

Para garantizar el adecuado servicio de la plataforma tecnológica en el ICC se analiza sistemáticamente el funcionamiento de los siguientes tres elementos: conectividad, disponibilidad de contenidos y herramientas informáticas de 
administración y gestión. En cuanto a la conectividad, que permite el acceso a Internet, lo ideal es disponer de dos proveedores para tener redundancia en el acceso, pero debido al alto costo que eso supone en el ICC se optó por contratar los servicios de una empresa proveedora de telefonía y telecomunicaciones que garantiza el 99.99\% de disponibilidad del servicio. La disponibilidad de los contenidos es la base del trabajo de los estudiantes, de allí su importancia. Para asegurar que los estudiantes pueden acceder las 24 horas y los 365 días del año a estos materiales, en el ICC los cursos se albergan en varios repositorios y servidores que operan en cluster. De modo que si llega a fallar algún elemento, la información siempre esté disponible en un servidor paralelo. Por su parte, las herramientas de software para la administración y gestión permiten prevenir posibles problemas en la red o en los servidores. Mediante su aplicación es posible conocer la velocidad y calidad de los sistemas conectividad, lo que facilita tomar acciones preventivas. Asimismo, permite conocer la capacidad almacenamiento disponible, la cantidad de memoria y de procesamiento utilizado y el número de usuarios conectados simultáneamente. Esta información permite, de ser el caso, aumentar los servicios y la memoria mediante la liberación de espacio. Este software permite además, en nuestro caso, verificar el funcionamiento de las Aulas Clavijero, mencionadas anteriormente, a las que acceden los estudiantes para emplear la infraestructura tecnológica y los servicios gratuitos de Internet. Por último, la calidad de la plataforma se asegura mediante la aplicación de software de protección de la red como los sistemas de antivirus y los sistemas de detección de intrusos.

\section{CONCLUSIONES}

En México la educación a distancia en línea está en sus inicios lo que se demuestra por una oferta de estudios superiores limitada y por el número reducido de estudiantes inscritos en esta modalidad, especialmente en el nivel de licenciatura. El aseguramiento de la calidad de esta oferta también apenas ha iniciado. Es de esperarse que conforme más instituciones participen en la evaluación externa de sus programas y hagan públicos los resultados obtenidos, se confirme el hecho de que los programas ofrecidos en esta modalidad poseen al menos una calidad equivalente a la de los programas escolarizados, si no es que mejor.

Existe una preocupación legítima del gobierno mexicano, de las asociaciones nacionales y de las instituciones educativas, así como de la sociedad en general, por asegurar que los estudios en la modalidad a distancia sean legales y de calidad reconocida. El riesgo de no hacerlo es enorme pues se pone en juego el futuro de 
miles de estudiantes que no tienen la opción de asistir a una institución tradicional para obtener una formación universitaria. Es necesario entonces normar y regular esta modalidad de estudios para evitar la proliferación de ofertas fraudulentas que se aprovechan de auténticas necesidades sociales y personales para ofrecer servicios educativos sin reconocimiento oficial y de una calidad discutible.

El modelo de aseguramiento de la calidad propuesto por el ICC es muestra de la responsabilidad social asumida y del compromiso que se tiene con los estudiantes para ofrecer programas educativos pertinentes, legales y que satisfacen estándares reconocidos de calidad en términos de sus programas y materiales de aprendizaje, de los docentes, de los servicios de apoyo académico, administrativo y tecnológico, así como de su infraestructura tecnológica. Los elementos que atiende guardan relación estrecha con los tres modelos de aseguramiento de la calidad de la educación a distancia revisados en este trabajo y las evidencias proporcionadas permiten afirmar que en el ICC se promueve la mejora continua, propósito último de cualquier esfuerzo de evaluación. En el ICC se lleva a cabo un seguimiento puntual y sistemático del funcionamiento de sus procesos académicos y administrativos con la intención de aprender de nuestro desempeño y tener claridad sobre las oportunidades de mejora, es decir, considerar al instituto como una institución que aprende (Senge, 1990).

Existe aún mucho por conocer de nuestros procesos. En particular, si bien se realiza un seguimiento del desempeño de los profesores, todavía es posible profundizar más en la calidad de la interacción sostenida entre ellos y sus estudiantes, así como de la calidad de la retroalimentación que ofrecen sobre sus trabajos. Asimismo, se reconoce que más debe hacerse para asegurar que los estudiantes que terminan sus estudios desarrollen las competencias profesionales esperadas. Un esfuerzo internacional en esta dirección es la iniciativa AHELO (por las siglas en inglés de Assessment of Higher Education Learning Outcomes) de la OCDE (OCDE, 2011), que pretende evaluar lo que los egresados saben hacer una vez que concluyen sus estudios superiores. El ICC estará atento para participar de dicha iniciativa una vez que se desarrollen los exámenes en las áreas atendidas por el instituto y se generalice su aplicación entre las instituciones miembros de la organización. Sin embargo, mientras esto ocurre, se deberá promover en el nuevo diseño de los cursos y entre los profesores la existencia de mayores y mejores oportunidades para que los estudiantes demuestren la adquisición de las competencias deseadas. Por último, un área desatendida aún en el ICC es el conocimiento de la calidad de la interacción sostenida entre los estudiantes. Es sabido que esta forma de interacción es uno de los pilares de la educación a distancia (Ally, op.cit.) y que promueve el aprendizaje colaborativo. 
La aplicación sistemática y continuada del modelo de aseguramiento de la calidad de los servicios educativos del ICC permitirá verificar su utilidad para fundamentar la toma de decisiones encaminada a la mejora continua de los servicios y consolidarlo como una herramienta eficaz para la rendición de cuentas.

\section{REFERENCIAS BIBLIOGRÁFICAS}

Allen, E.; Seaman, J. (2010). Learning on Demand. Online Education in the United States 2009. [en línea]. Disponible en: http://sloanconsortium. org/publications/survey/pdf/ learningondemand.pdf (consulta 2011, 6 de mayo).

Ally, M. (2004). Foundations of Educational Theory for Online Learning. En Anderson, T.; Elloumi, F. (Eds.). Theory and Practice of Online Learning. Athabasca: Athabasca University.

Ander-Egg, E. (1999). Diccionario de Pedagogía. Buenos Aires: Magisterio del Río de la Plata.

Anderson, T. (2004). Toward a Theory of Online Learning. En Anderson, T. y Elloumi, F. (Eds.). Theory and Practice of Online Learning. Athabasca: Athabasca University.

Asociación Nacional de Instituciones de Educación Superior (2011). Directorio Nacional de Instituciones de Educación Superior. [en línea]. Disponible en: www. anuies.mx/la anuies/diries (consulta 2011, 2 de mayo).

Athabasca University (2010). Annual report to Alberta Advanced Education and Technology. [en línea]. Disponible en: http://www2.athabascau.ca/aboutau/ documents/annual/report2010.pdf (consulta 2011, 6 de mayo).

Banco Mundial (2011). Internet users per 100 people. [en línea]. Disponible en: http:// data.worldbank.org/indicator/IT.NET. USER.P2?order=wbapi data value 2009+wbapi data value+wbapi data value-last\&sort=desc (consulta 2011, 13 de mayo).

Bekele, T. A. (2010). Motivation and Satisfaction in Internet-Supported Learning Environments. Review Educational Technology \& Society, 13 (2), (116-127). [en línea]. Disponible en: http://www.ifets.info/journals/13 2/10.pdf (consulta 2010, $2 \mathrm{de}$ diciembre).

Bollinger, D.; Martindale, T. (2004). Key Factors for Determining Student Satisfaction in Online Courses. [en línea]. Disponible en: http://teachable. org/papers/2004 satisfaction factors. pdf (consulta 2011, 10 de mayo).

Brennan, J.; Williams, R. (2009). Collecting and using student feedback on quality and standards of learning and teaching in higher education. Report to HEFCE. [en línea]. Disponible en: http://www.hefce. ac.uk/pubs/rdreports/2003/rdo8 03 (consulta 2011, 4 de abril).

Centro Virtual para el Desarrollo de Estándares de Calidad para la Educación a Distancia en América Latina y el Caribe (2004). Memoria. Estándares de Calidad. Loja: UTPL.

Chickering, A.; Ehrman, S. (1996). Implementing the Seven Principles: Technology as Lever. American Association for Higher Education Bulletin, 49 (2), (3-6).

Comités Interinstitucionales para la Evaluación de la Educación Superior 
(2010). Metodología General para la Evaluación de Programas Educativos a Distancia. México, D.F.: CIEES.

Council for Higher Education Accreditation (2002). Accreditation and Assuring Quality in Distance Learning. Institute for Research and Study of Accreditation and Quality Assurance, Council for Higher Education Accreditation. [en línea].Disponibleen:http://www.ncahlc. org/information-for-institutions/ publications.html. (consulta 2011, 29 de abril).

Dela Garza, J. (2011). Evaluación Diagnóstica. Estrategia para Elevar la Calidad de educación Superior a Distancia. 3er Congreso Virtual Educa México, 16-18 de marzo 2011, Chiapas, México. [en línea]. Disponible en: http://libroselectronicos. unach.mx/virtual11/javier/index.html (consulta 2011, 11 de mayo).

Diaz, D. (2002). Online Drop Revisited. [en línea]. Disponible en: http:// technologysource.org/article/online drop rates revisited// consulta 2011, 13 de mayo).

Facundo, A. H. (2004). Tecnologías de la Información y Comunicación y Educación Superior Virtual en Latinoamérica y el Caribe. Evolución, Características y Perspectivas. Bogotá: UNESCO/CRESALC.

Fahy, P. (2004). Media Characteristics and Online Learning Technology. En Anderson, T. y Elloumi, F. (Eds.). Theory and Prectice on Online Learning. Athabasca: Athabasca University.

Gaskell, A. (2009). Student satisfaction and retention: are they connected? Open Learning (Vol. 24) [en línea]. Disponible en: $\quad$ http://pdfserve.informaworld. com/149352 915021385.pdf (consulta 2010, 2 de diciembre).

Instituto Internacional de la Unesco para la Educación Superior de América latina y el Caribe (2006). Informe sobre la
Educación Superior en América latina y el Caribe 200O-2005. Caracas: IESALC

Instituto Nacional de Geografía y Estadística (2010). Encuesta de Hogares sobre Disponibilidad y Uso de Tecnologías de la Información. [en línea]. Disponible en: http://www.consulta.mx/Estudio. aspx?Estudio=tecnologa-internet-inegi (consulta 2011, 6 de mayo).

Indhira Gandhi National Open University (2010). Building an Inclusive Knowledge Society, 2010 Profile. [en línea]. Disponible en: www.ignou.ac.in (consulta 2010, 5 de mayo).

Mercado, R. (2007) (Coord.). Guía para el Diseño de Cursos en Línea. Xalapa: Universidad Veracruzana.

OCDE (2001). Education Policy Analysis. Paris: OECD.

OCDE (2011). Testing Student and University Performance Globally: OECD's AHELO. [en línea]. Disponible en: http://www. oecd.org/document/22/0,3746, en

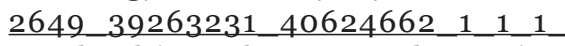
1,00.html (consulta 2011, 12 de mayo).

OECD (2008). Tertiary Education for the Knowledge Society. OECD Thematic Review of Tertiary Education.Synthesis Report. Paris: OCDE.

Open University (2010). Annual Report. [en línea]. Disponible en: http://www.open. ac.uk/about/documents/about-annualreport-2010.pdf (consulta 2011, 5 de mayo).

Open Univesities Australia (2010). Annual Report 2010. [en línea]. Disponible en: https://www.open.edu.au/public/file/ OUA Annual Report 2010.pdf?id=ca4 5fb804688e23eb739bfd19325056c\&CA CHEID $=$ ca $45 \mathrm{fb} 804688$ e23eb739bfd 193 25056c (consulta 2011, 6 de mayo).

Ortiz. A. (2003). Estudio sobre el Uso de las Tecnologías de la Comunicación e Información para la Virtualización de la Educación Superior en México. IESALC/ANUIES. 
Palmer, S. R. \& Holt, D. M. (2009). Examining student satisfaction with wholly online learning. Journal of Computer Assisted Learning , 25, (101-113).

Rama, C. (2007). La educación Virtual en los Posgrados: Una Nueva Relación entre el Escenario Global y los Ámbitos Nacionales. En: Mena, M. (Coord.). Construyendo la Nueva Agenda de la Educación a Distancia. Buenos Aires: La Crujía.

Robles, H. (2006). La Economía Basada en el Conocimiento. Las Condiciones de los EstadosMexicanos.[enlínea].Disponible en: http://www.razonypalabra.org.mx/ anteriores/n49/bienal/Mesa\%2012/ HectorRobles.pdf (consulta 2011, 12 de mayo).

Senge, P. (1990). The Fifth Discipline. The Art and Practice of the Learning Organization. New York: Double day

Shieh, R. S.; Gummer, E.; Niess, M. (2008). The Quality of a Web-Based Course: Perspectives of the instructor and the students. TechTrends, (61-68).

Sloan Consortium (2008). Staying the Course. Online Education in the United States. [en línea]. Disponible en: www. sloanconsortium.org/sites/default/files/ staying the course-2.pdf (consulta 2010, 5 de mayo).

Strachiota, E. (2006). The Use of Survey Research to Measure Student Satisfaction in Online Courses. [en línea]. Disponible en: http://www. umsl.edu/continuinged/education/ mwr2po6/pdfs/D/Strachota Use of Survey Research.pdf (consulta 2011, 10 de mayo).

The Open Univesity of China (2010). Annual Report. [en línea]. Disponible en: http://en.crtvu.edu.cn/about/annualreport/2010 (consulta 2011, 6 de mayo).

Tuirán, R. (2011). La educación superior en México: avances, rezagos y retos. http://ses.sep.gob.mx/wb/ses/la educacion superior en mexico avances rezagos y retos (consulta 2011, 9 de mayo).

UNESCO (2009). Compendio Mundial de la Educación 2009. Comparación de las Estadísticas de Educación en el Mundo. [en línea]. Disponible en: http:// www.uis.unesco.org/template/pdf/ ged/2009/GED 2009 SP.pdf (consulta 2011, 9 de mayo).

Universidad Nacional a Distancia (2010). Memoria Anual Curso 2009-10. [en línea]. Disponible en: http://portal. uned.es/pls/portal/docs/PAGE/ UNED MAIN/LAUNIVERSIDAD/ VICERRECTORADOS/SECRETARIA/ PORTLET/MEMORIA\%2OANUAL\%20 UNED\%202009-10.PDF (consulta 2011, 13 de mayo).

University of South Africa (2010). University of South Africa Annual Report 2009. [en línea]. Disponible en: http://www.unisa. ac.za/happening/docs/AnnualReport 2009.pdf (consulta 2011, 13 de mayo).

\section{PERFIL ACADÉMICO Y PROFESIONAL DE LOS AUTORES}

Ricardo Mercado del Collado. Licenciado, maestro y doctor en Psicología. En la Subsecretaría de Educación Superior e Investigación Científica fue Director de Desarrollo Universitario y Secretario Adjunto de la Comisión Nacional de Evaluación de la Educación Superior, CONAEVA. En la Universidad Veracruzana fue Director de 
Planeación Universitaria y Director General de la Universidad Veracruzana Virtual. Actualmente es Subdirector Académico del Instituto Consorcio Clavijero.

E-mail: rmercado@clavijero.edu.mx

Mónica López Granados. Licenciada en Ciencias de la Comunicación por la Universidad Autónoma Metropolitana-Xochimilco, Maestra en Pedagogía por la Johann Wolfgang Goethe Universität (Frankfurt am Main, Alemania) y especialista en la creación y valoración de espacios educativos virtuales desde hace 15 años. Ha laborado en la Universidad Virtual del Tec de Monterrey, Universidad Tecnológica de México y en el Instituto Consorcio Clavijero.

E-mail: molopez@clavijero.edu.mx

Gustavo Manuel Balderas Rosas. Subdirector de Planeación, evaluación y desarrollo Institucional del Instituto Consorcio Clavijero. Maestría en sistemas de información, 29 años de experiencia docente en la Universidad Veracruzana, ex director de redes e infraestructura tecnológica de la Universidad Veracruzana, ex subdirector de tecnológicas de la Información de la Secretaria de Educación de Veracruz, con amplia experiencia en implementación de proyectos de redes y sistemas de información, además de un diplomado en Política y Gestión Educativa.

E-mail: gbalderas@clavijero.edu.mx

\section{DIRECCIÓN DE LOS AUTORES:}

Instituto Consorcio Clavijero

Santiago Bonilla 103,

Colonia Obrero Campesina

C.P. 91020

Xalapa, Veracruz, México

Fechas de recepción del artículo: 06/07/11

Fecha de aceptación del artículo: 30/07/11

\section{Como citar este artículo:}

Mercado del Collado, R.; López Granados, M.; Balderas Rosas, G. (2011). El aseguramiento de la calidad en el Instituto Consorcio Clavijero. RIED. Revista Iberoamericana de Educación a Distancia, volumen 14, $\mathrm{n}^{0}$ 2, pp. 15-47. 